\title{
ELEMENTAL ANALYSIS OF PALLADIUM ELECTRODES AFTER PD/PD LIGHT WATER CRITICAL ELECTROLYSIS
}

\author{
YU TORIYABE \\ Division of Quantum Science and Engineering, Graduate School of Engineering, \\ Hokkaido University \\ North 13, West 8, Kita-ku, Sapporo, 060-8628, Japan \\ torigoya@pop.qe.eng.hokudai.ac.jp \\ TADAHIKO MIZUNO \\ Division of Energy and Environmental System, Graduate School of Engineering, \\ Hokkaido University \\ North 13, West 8, Kita-ku, Sapporo, 060-8628, Japan \\ TADAYOSHI OHMORI \\ Hokkaido Institute of Technology, \\ Maeda, Teine-ku, Sapporo 006-8585, Japan \\ YOSHIAKI AOKI \\ Technology and Electronics College of Hokkaido, \\ Nakanoshima, Toyohira-ku, Sapporo 062-0922, Japan
}

\begin{abstract}
Elemental analyses of palladium electrodes were conducted after a new type of light water electrolysis was performed at optimum conditions in a system designed to induce a nuclear reaction. This process is referred to as $\mathrm{Pd} / \mathrm{Pd}$ light water critical electrolysis. The conjecture that a nuclear transmutation process is occurring in this experiment is easier to test in this system, because it is easy to determine whether the elements detected on the cathode surface are impurities or transmutation products. We assume that the elements found only on the cathode surface, and nowhere else in the cell as contamination, namely iron, titanium, chromium and so on, must be transmutation products. Furthermore, countless Ohmori-type palladium craters were observed for the first time for this system, and these are evidence that nuclear reactions occurred at the electrode surface.
\end{abstract}

\section{Introduction}

Since Ohmori et al. reported nuclear transmutation reactions with anomalous isotopic yields in a light water electrolysis system [1-4], many researchers have claimed various kinds of low energy nuclear reactions. At the same time they observed continuous excess heat as results and numerous craters as an evidence of nuclear reactions. These results in the light water system deserve close attention because they strongly suggest the existence of condensed matter nuclear reactions.

It is, however, still difficult to replicate that reaction in normal electrolysis. Through our previous experiments and in our other reports [5-8], we conjecture that the 
electrolysis system and electrical parameters are very important factors in inducing nuclear reactions. We have investigated an optimum electrolysis system and conditions and describe details in this paper.

Both transmutation products and surface damage are needed to prove that a nuclear reaction has occurred. Figure 1 shows an example of damage on a gold electrode reported by Ohmori et al. [9]. Although many researchers have reported nuclear transmutations, as far as we know, there has been no other reports of the Ohmori-type craters. If nuclear reactions occur on the electrode surface, surface damage like Ohmori-type craters should be found after the experiment.
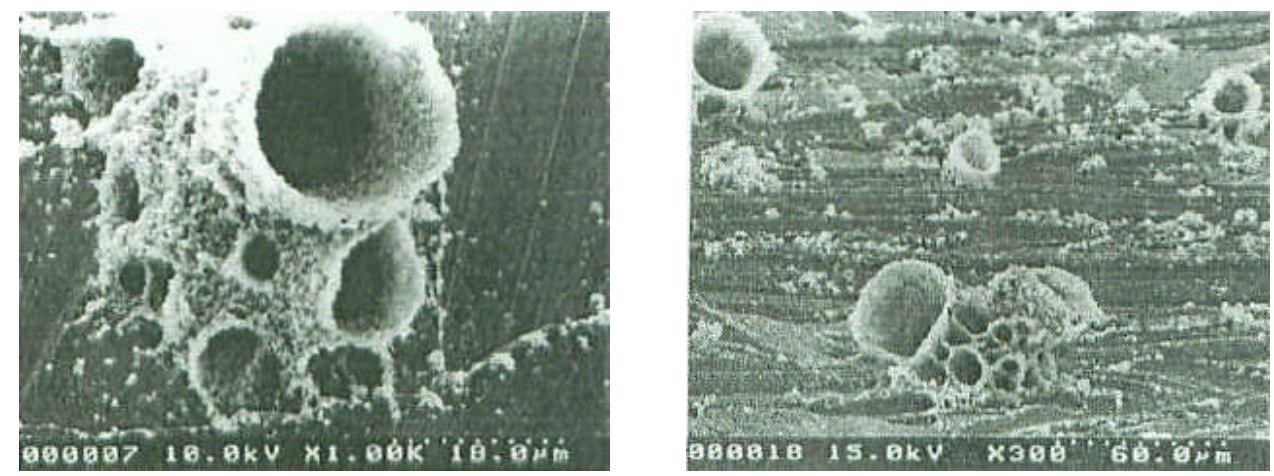

Figure 1. Surface damage as indirect evidence of nuclear reactions occurred at the electrode surface. These craters were observed on gold electrode after normal electrolysis, with current density of $0.5 \mathrm{~A} / \mathrm{cm}^{2}$ for 30 days in $0.5 \mathrm{M} \mathrm{Na}_{2} \mathrm{CO}_{3}$ solution [9].

\section{Plasma Electrolysis}

\subsection{Time Variations}

Plasma electrolysis can produce a large amount of excess heat, and nuclear transmutation products. When the temperature of an electrode exceeds the boiling temperature of electrolyte due to intense polarization, a thin vapor layer is generated at the electrode / electrolyte interface in which high electric field ionize vapor molecules to generate the plasma state. Formation of this vapor film is the first key factor to achieve the plasma electrolysis condition.

Time variations of cell voltage and current during the plasma electrolysis are shown in Figure 2. In this case the axis of ordinates indicates not only current, but also current density, because the electrode surface of cathode is ca. $1 \mathrm{~cm}^{2}$. The period of electrolysis is divided into 6 regions for the sake of convenience, namely: conventional region, critical region, breakdown point, transitional region, partial plasma region, and full plasma region.

The electrical current increases with applied voltage, up to the breakdown point, at which time a sheath of vapor film is generated. Then the current drops down through the 
transitional region, because the electrode and the electrolyte cannot touch each other directly owing to the vapor layer.

If cell voltage is sufficiently high, some atoms or molecules in that gas phase are ionized. Therefore current does not drop to zero, and the electrode temperature remains high due to the good electrical conductivity and reduced thermal loss of the gas phase. Starting from this region, light emission occurs. The color of the light depends on the electrolyte solution. The light emission area expands with increasing cell voltage. Finally, in region-6, the glow covers the entire surface of the electrode and the intensity of light becomes strong. We defined these two regions, region-5 and 6 , as the plasma region.

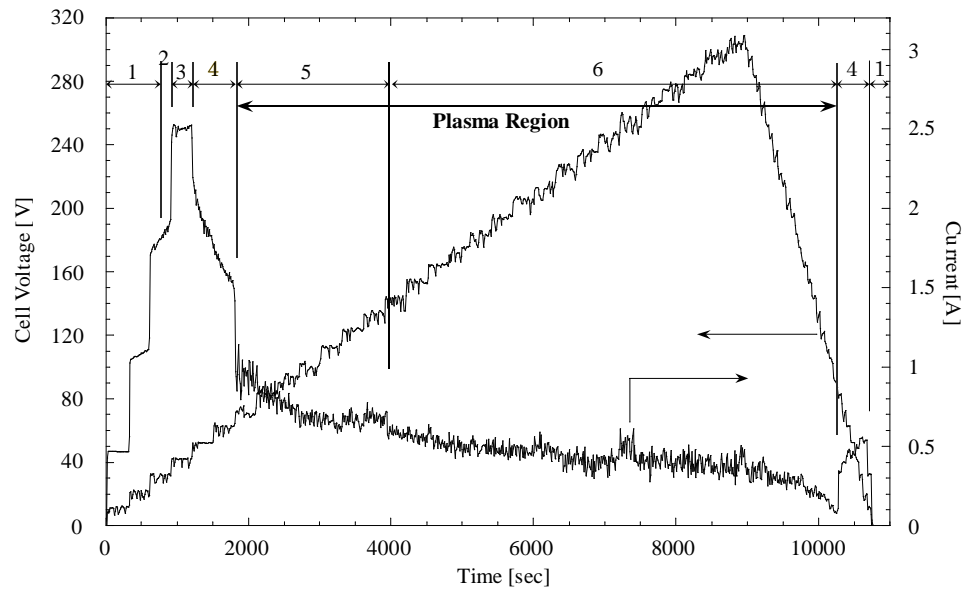

Figure 2. Time variations of cell voltage and current during plasma electrolysis with $1.5 \mathrm{~mm}$ diameter tungsten cathode in $0.2 \mathrm{~mol} / \mathrm{dm}^{3} \mathrm{~K}_{2} \mathrm{CO}_{3}$ solution. Six regions are identified: 1 . Normal; 2. Critical; 3. Breakdown point; 4. Transitional; 5. Partial plasma; 6. Full plasma.

\subsection{Relation}

A voltage-current relation of plasma electrolysis is shown in Figure 3, which is converted from Figure 2. When current density increases above approximately $2.5 \mathrm{~A} / \mathrm{cm}^{2}$, the type of electrolysis changes to plasma electrolysis where the current is almost constant. This critical value also supports the experimental result reported by Ohmori et al. [10]. It is necessary to select an experimental condition, current and voltage from this VI curve. 


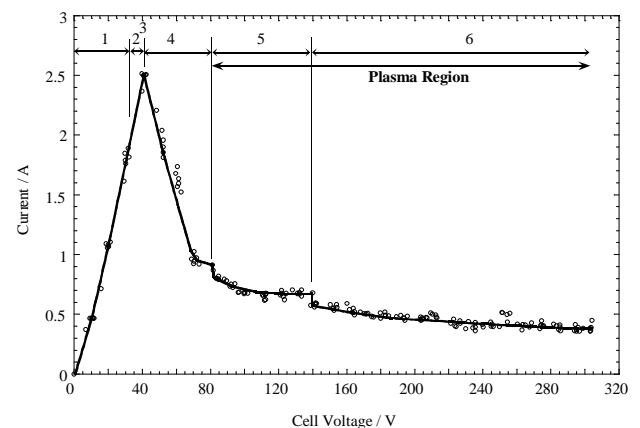

Figure 3. Voltage-current relation during plasma electrolysis converted from Fig.1.

\subsection{Shortcomings}

Although plasma electrolysis can induce a large amount of excess heat with hydrogen and anomalous nuclear transmutation [11, 12], this type of electrolysis has some critical shortcomings.

At the boundary between region- 5 and 6 , that is, at the time when whole area of the electrode surface is covered with the glow discharge, the temperature of the surface rises to $1,400^{\circ} \mathrm{C}$. Furthermore, the local temperature exceeds the melting point of the electrode at the spark-like arc discharges that occur due to current flow paths between the electrode and the electrolyte. Therefore the electrode cannot survive more than $30 \mathrm{~min}$ without being melted or broken. The electrolyte solution is also decomposed by pyrolysis from the high temperature plasma.

The most serious problem is that the rare nuclear transmutation products on the electrode surface are lost from this damage. Therefore another, milder type of electrolysis, which does not severely damage or disintegrate the electrode, is called for to prove that nuclear reactions occur.

\section{Critical Electrolysis}

\subsection{Optimum Region}

We assume the positive correlation between the excess heat and current density exists in normal region, while the excess heat increases with the input voltage in the plasma region. This assumption is illustrated in Figure 4 with the VI relation described in Figure 3. In fact, some researchers have reported these correlations [13-15].

Since the plasma region is destructive, Ohmori et al. specified that region-2 is the most favorable one to obtain excess heat and transmutations, and they referred to this type of electrolysis as Critical Electrolysis [7, 8]. Although the exact conditions required depend on the experimental system, a target current density is approximately $2.5 \mathrm{~A} / \mathrm{cm}^{2}$. With this type of electrolysis, a long duration experiment is possible because the electrode 
is not damaged. Thus transmutation products are well preserved and continuous excess heat can be obtained.

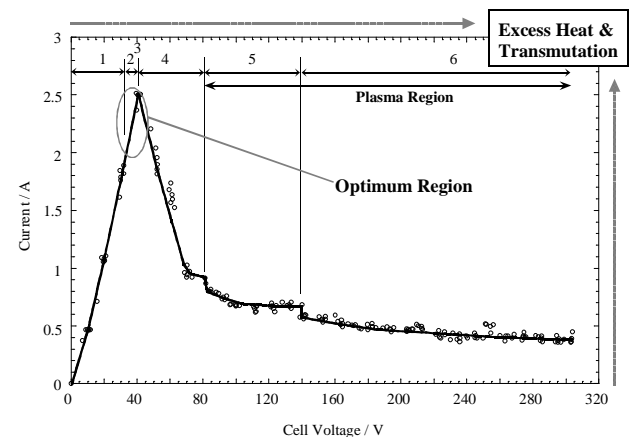

Figure 4. An optimum region to induce nuclear reaction without disintegrating the electrode.

\subsection{Results}

We conducted critical electrolyses with nickel cathodes in $1 \mathrm{M} \mathrm{Na}_{2} \mathrm{CO}_{3}$ solutions [5]. Figure 5 shows EDX spectrums of nickel electrodes after the experiment through which the current density was kept ca. $2.6 \mathrm{~A} / \mathrm{cm}^{2}$ for 15 days. The mean voltage applied was $14 \mathrm{~V}$ and solution temperature went up to $80^{\circ} \mathrm{C}$.

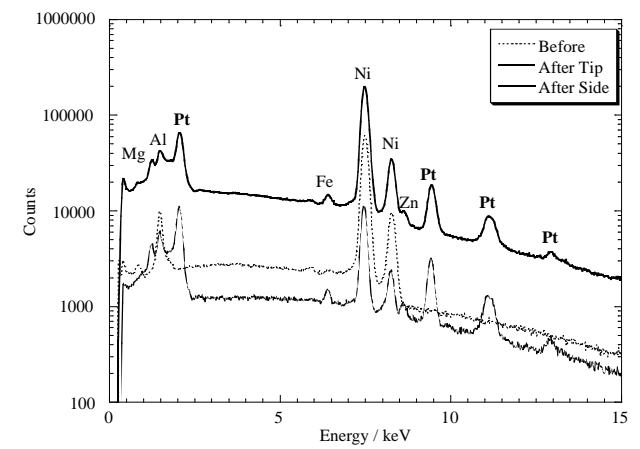

Figure 5. EDX spectrums of nickel electrodes before and after the critical electrolysis with current density of $2.6 \mathrm{~A} / \mathrm{cm}^{2}$ for 15 days in $1 \mathrm{M} \mathrm{Na}_{2} \mathrm{CO}_{3}$ solution.

The strong platinum peaks we detected probably originated from the counter electrode, although the isotopic ratio for this platinum has not been confirmed yet. In general, the platinum anode is less dissolved and electrodeposited in an alkaline solution, especially $\mathrm{K}_{2} \mathrm{CO}_{3}$ or $\mathrm{Na}_{2} \mathrm{CO}_{3}$. However the experimental results suggest that this unusual reaction is accelerated in certain conditions, and this platinum complicates transmutation processes such as photofission [16]. The electrodeposited platinum makes an evaluation of the process difficult. Therefore in the experimental system the anode material should be the same as the cathode. 


\section{$4 \quad$ Pd/Pd Critical Electrolysis}

\subsection{Experimental Set-up}

$\mathrm{Pd} / \mathrm{Pd}$ Critical Electrolysis is defined as the optimum condition to evaluate the transmutation process precisely. In this system, the effect of materials electrodeposited from the anode is avoided by making the anode from the same element as the cathode. This limits the elements detected on the cathode to being either impurities from elsewhere in the cell, or from transmutation. Furthermore, if these elements are transmutation products, evaluating them is simpler.

Figure 6 shows a schematic view of an experimental set-up. The system should be very simple, because it has to be cleaned very carefully before the experiment, to avoid contamination.

A Teflon (PFA) cell (Flon Industry Co., Ltd.) is used. The cell volume is $300 \mathrm{cc}$, diameter $80 \mathrm{~mm}$, height $70 \mathrm{~mm}$. The cell is capped with silicon rubber. Although water vapor cannot leak from this system, hydrogen can. It easily passes from a hole used to replenish decomposed electrolyte with Milli-Q purified water, even though this hole is stopped with silicon rubber.

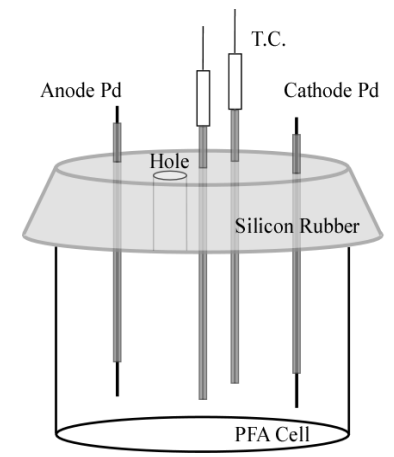

Figure 6. The experimental set-up for the Pd/Pd critical electrolysis experiments.

In this experiment, the anode and cathode are made from identical palladium wires taken from the same stock. Both are $99.95 \%$ pure Pd, $1.0 \mathrm{~mm}$ diameter, $15.7 \mathrm{~mm}$ long, with ca. $0.5 \mathrm{~cm}^{2}$ surface area. They were polished with two kinds of emery paper (\#1500 and \#2000), and washed with acetone. After being covered with Teflon (PTFE) tubes, they were located at the both side of the cell symmetrically. Before the experiment, the cell and electrodes were cleaned carefully with nitric acid, or sometimes with mixed acid $\left(1: 1 \mathrm{H}_{2} \mathrm{SO}_{4}+\mathrm{HNO}_{3}\right)$, and rinsed with Milli-Q water.

The electrolyte solution of $1 \mathrm{M}$ and $200 \mathrm{cc}$ was prepared by $\mathrm{K}_{2} \mathrm{CO}_{3}$ (Kanto Chemical Co., Inc.) whose purity is over $99.95 \%$ and Milli-Q water with specific resistance over $18.0 \mathrm{M} \Omega \cdot \mathrm{cm}$. The isotopic abundance of hydrogen and deuterium atoms of this Milli-Q 
water is natural, since ordinary water was used. The natural concentration of $\mathrm{D}_{2} \mathrm{O}$ is so low the influence from it is negligible.

The cell was placed in a constant temperature chamber (MIR-151, Sanyo Electric Co., Ltd.). The solution temperature was roughly measured by two thermocouples, which are located at the center and edge of the cell. Air temperature was also monitored and kept 22.0 24. $0^{\circ} \mathrm{C}$.

The current density exceeded $2.5 \mathrm{~A} / \mathrm{cm}^{2}$. It was controlled by a constant current/voltage power supply (GP0250-3R, Takasago Ltd.). The experiments were continued for 7 or 10 days, while various data was collected on a data logger (8421-50, Hioki E. E. Co.). During the electrolysis, Milli-Q makeup water was added every 12 hours to restore the volume of electrolyte lost to decomposition.

\subsection{Elemental Analysis}

After the experiments both electrodes were observed with an SEM (JSM-6500F, JEOL Ltd.) and analyzed by EDX. Figure 7 shows the spectra from the whole area of each electrode. In this case, current density had been $3.2 \mathrm{~A} / \mathrm{cm}^{2}$ for 7 days. The thick line indicates the cathode palladium spectrum, and the thin line is the anode spectrum. The count of the anode spectrum is multiplied by 1.18 to make it overlap with the cathode spectrum. (Although a before-and-after comparison of spectra is common in these experiments, in this case a comparison of the anode and cathode is more suitable.)
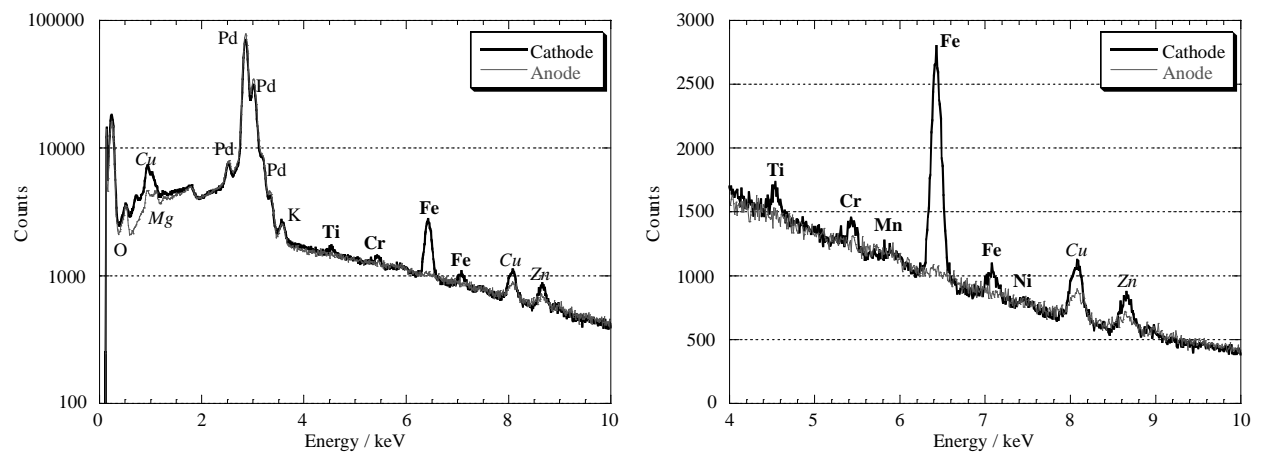

Figure 7. EDX spectrums of palladium electrodes, cathode and anode, after the $\mathrm{Pd} / \mathrm{Pd}$ critical electrolysis with current density of $3.2 \mathrm{~A} / \mathrm{cm}^{2}$ for 7 days in $1 \mathrm{M} \mathrm{K}_{2} \mathrm{CO}_{3}$ solution. Counts of anode spectrum are multiplied 1.18 to be overlapped with the cathode spectrum.

The elements shown in bold letters, iron, titanium, chromium, manganese and nickel, were detected in the cathode palladium only. On the other hand, the elements shown in italic letters, copper, zinc and magnesium, were detected in both electrodes. None of the elements listed here were detected in the samples before the experiment. Therefore we assume that elements shown in bold letters must be transmutation products. The iron peaks especially were detected in all cathode samples after $\mathrm{Pd} / \mathrm{Pd}$ critical electrolysis.

The elements shown in italics may have originated from two possible sources: transmutations or impurities. If these were transmutation products, at least two processes 
may explain their existence. The first nuclear reaction presumably occurred at the cathode and only produced the bold letter elements, while the second reaction occurred at both electrodes, and produced the italic letter elements.

Since we have not analyzed the samples by the other methods, we cannot determine the origin of these elements yet. If we confirm the isotopic distribution is anomalous, we will then be prepared to discuss the origin and transmutation processes of the detected elements, whether they are electrodeposited impurities or transmutations.

\subsection{Micro Structure}

Figure 8 and 9 are SEM photographs of the anode palladium surfaces and the cathode surfaces after the $\mathrm{Pd} / \mathrm{Pd}$ critical electrolysis, respectively. The anode palladium has some cracks due to the oxidation.

On the cathode surfaces, countless Ohmori-type palladium craters were observed. This is indirect evidence of nuclear reactions. These craters, which are located along the surface cracks or grain boundaries, were observed on the cathode only up until now. This result indicates that the nuclear reactions occurs more easily around the surface and the cracks.

The maximum width and height of craters are over 10 micrometers. These crater sizes have a positive correlation with the current density. This result supports our prediction that the amount of excess heat and transmutation have a positive correlation with increase of current density. Therefore the critical region is optimum to induce the nuclear reactions as described above.
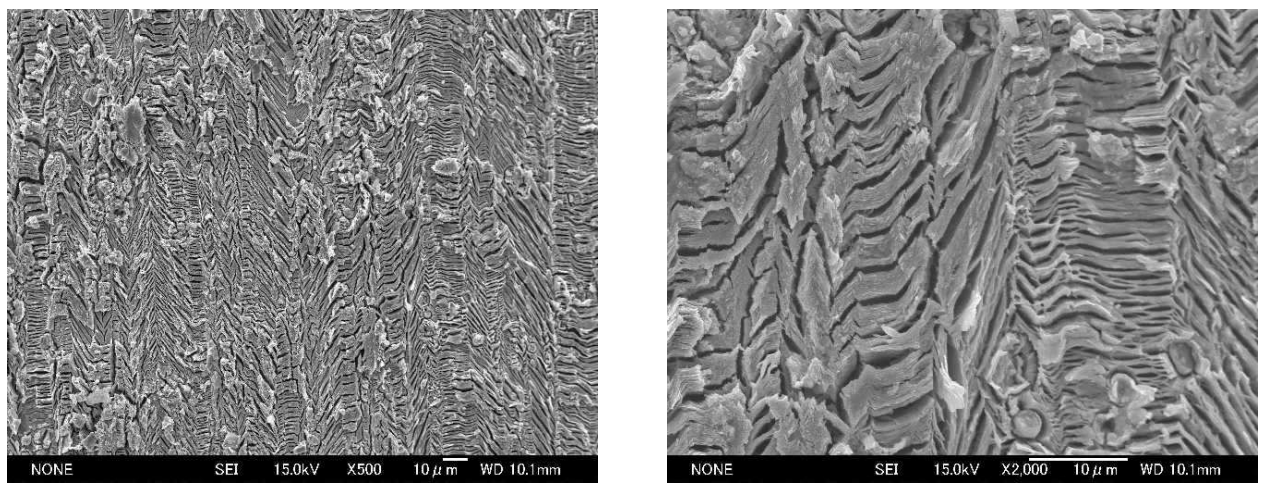

Figure 8. SEM photographs of the anode palladium surfaces after $\mathrm{Pd} / \mathrm{Pd}$ critical electrolysis with current density of $3.6 \mathrm{~A} / \mathrm{cm}^{2}$, for 10 days in $1 \mathrm{M} \mathrm{K}_{2} \mathrm{CO}_{3}$ solution. The magnifying power and standard scale lines are shown at the bottom of each photograph. 

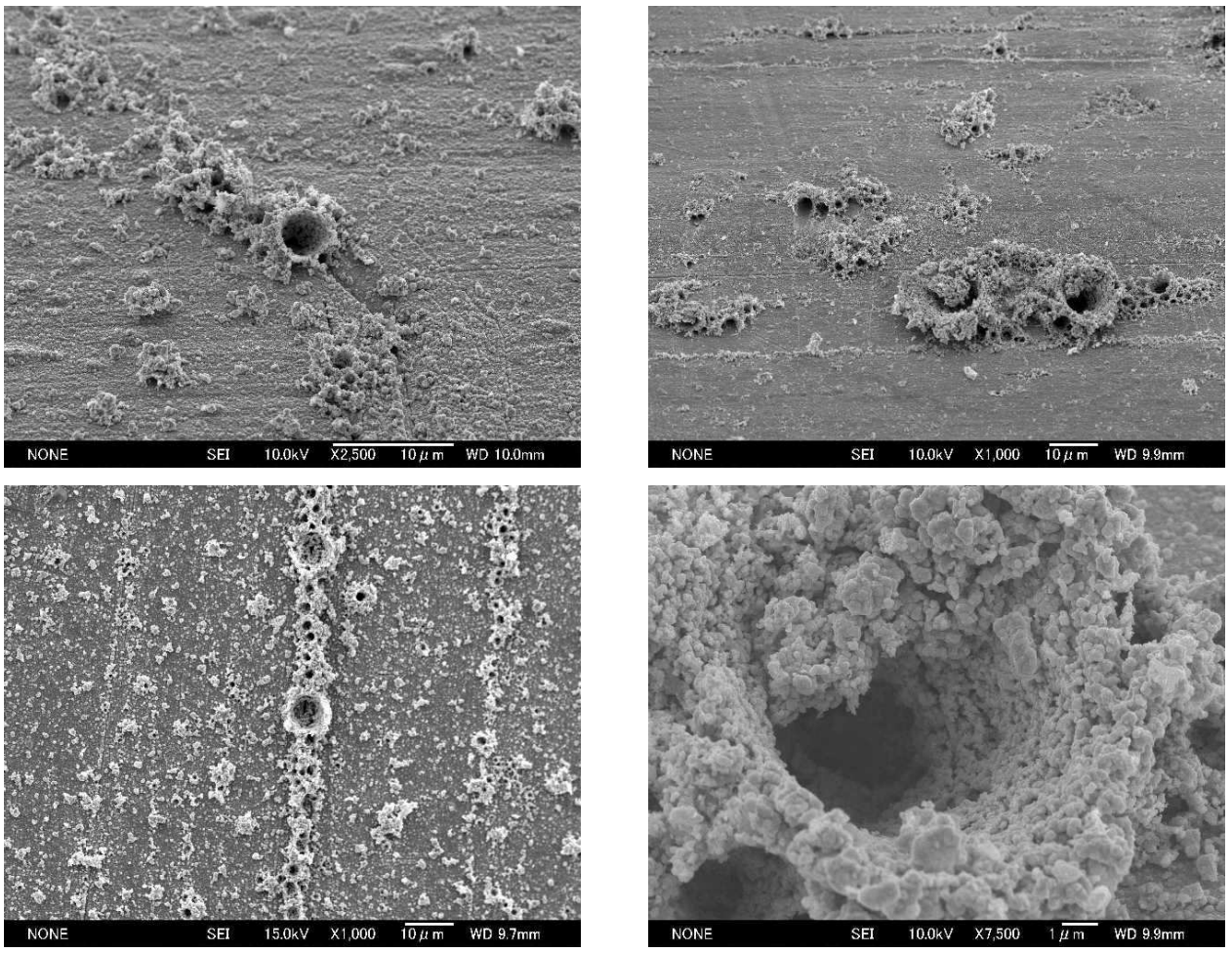

Figure 9. SEM photographs of Ohmori-type palladium craters on the cathode surfaces after Pd/Pd critical electrolysis with current density of $3.6 \mathrm{~A} / \mathrm{cm}^{2}$, for 10days in $1 \mathrm{M} \mathrm{K}_{2} \mathrm{CO}_{3}$ solution. Magnifying power and standard scale lines are shown at the bottom of each photograph. The craters whose maximum size is over 10 micrometers are located along the surface cracks.

\subsection{Discussion}

To our knowledge, this is the first report of Ohmori-type craters observed on palladium electrodes. The characteristics of these palladium craters are similar to the gold craters first reported by Ohmori et al. [3]. Although the report was made 10 years ago, the formation mechanism is still unknown.

Kamada et al. reported anomalous heat evolution and surface melting of deuteron implanted aluminum foil upon electron bombardment [17]. They estimated that the amount of anomalous heat exceeds the total amount from any kind of chemical reaction and, therefore, concluded that a novel nuclear reaction must have occurred in the deuterated aluminum. If the palladium electrode was melted due to this type of nuclear reaction, palladium gas should have erupted. Then it should have cooled down in the solution to be recrystallized like the crater.

Numata $e t$ al. found vortex patterns on well-annealed, thick palladium electrodes after long term heavy water electrolysis [18], and simulated a magnetic interaction of hypothetical particles to elucidate an evolution mechanism of vortexes [19]. They showed 
the vortex can be formed at the electrode/electrolyte interface by the FEM method. If charged palladium particles dissolved from the anode were caught in a vortex which is generated at the electrode/electrolyte interface, they might accumulate to form the cylindrical shape like the vortex to form the craters.

Mizuno et al. described a large explosion that occurred during a normal light water electrolysis [20, 21]. They made a rough estimate of the energy balance and concluded that it could not be a simple explosion, but rather it must have been caused by a large burst of anomalous heat. If micro explosions occurred on the electrode surface from this same type of heat, craters could be generated.

It is still difficult to explain the mechanism by these theories. Although they may be impurities electrodeposited uniformly, we claim that the crater is indirect evidence of nuclear reaction occurring at the electrode surface.

\section{Conclusion}

$\mathrm{Pd} / \mathrm{Pd}$ light water critical electrolysis was performed at the optimum conditions and system to induce nuclear reactions. In this study the shape of both electrodes was exact; $\mathrm{u}$ just same and the current density was over $2.5 \mathrm{~A} / \mathrm{cm}^{2}$, for 7 or 10 days, in $1 \mathrm{M} \mathrm{K}_{2} \mathrm{CO}_{3}$ solution whose temperature was up to $70 \sim 90^{\circ} \mathrm{C}$.

After the experiment, the obvious transmutation products namely iron, titanium, chromium, manganese, nickel were detected by EDX. In particular, the iron peaks are very strong and detected on all cathode samples. The anomalous isotopic yield of detected iron is expected since the anomaly has already claimed by many researchers [3, 4, 22]. The elements detected from the both electrodes, namely, cupper, zinc and magnesium, however, cannot indicate their origin at this time. If they are transmutation products, at least two mechanisms exist.

The analysis of isotopic yields for all elements detected by SIMS is indispensable to prove nuclear reactions. Quantitative analysis of the detected elements, distribution especially around craters, and isotopic yields are under considerations. Moreover, nuclear radiation detection could give important information to evaluate the process. Precise heat measurement is also required. Accurate estimation and reduction of impurities is also vital.

Although the isotopic yields have not yet been confirmed in this study, Ohmori et al. already reported anomalous isotopic distribution of palladium with excess heat in their $\mathrm{Pd} / \mathrm{Pt}$ critical electrolysis system [7, 8]. Furthermore the indirect evidence of nuclear reactions, namely Ohmori-type palladium craters were observed for the first time. Since the transmutation products and the surface damage have been found, $\mathrm{Pd} / \mathrm{Pd}$ critical electrolysis is considered optimum to induce condensed matter nuclear reactions. 


\section{References}

1. T. Ohmori and M. Enyo, Proceedings of ICCF4, Vol.1, N2.3 (1993)

2. T. Ohmori and M. Enyo, J. New Energy, Vol.1, No.1, 15 (1996)

3. T. Ohmori, T. Mizuno and M. Enyo, J. New Energy, Vol.1, No.15, 90 (1996)

4. T. Ohmori, M. Enyo, T. Mizuno, Y. Nodasaka and H. Minagawa, Fusion Technol., 31, 210 (1997)

5. Y. Toriyabe, T. Mizuno, T. Ohmori and Y. Aoki, Proceedings of JCF6, 11 (2005)

6. T. Mizuno, T. Ohmori and T. Akimoto, Proceedings of ICCF10 (2003)

7. T. Ohmori, S. Narita, H. Yamada, T. Mizuno and Y. Aoki, Proceedings of JCF4, 22 (2003)

8. T. Ohmori, T. Mizuno, H. Yamada and S. Narita, Proceedings of JCF5, 36 (2004)

9. A. Takahashi, H. Numata, H. Yamada, Y. Iwamura, T. Ohmori, T. Mizuno and T. Akimoto, Study of the nuclear reactions in the solid, 159 (1999)

10. T. Ohmori, Current Topics in Electrochemistry, 7, 102 (2000)

11. T. Mizuno, T. Ohmori, T. Akimoto and A. Takahashi, Jpn. J. Appl. Phys., 39, 6055 (2000)

12. T. Mizuno, T. Akimoto, K. Azumi, T. Ohmori, Y. Aoki and A. Takahashi, Jpn. J. Appl. Phys., Vol. 44, No. 1A, 396 (2005)

13. T. Ohmori and T. Mizuno, Current Topics in Electrochemistry, 5, 37 (1997)

14. M. C. H. McKubre, S. Crouch-Baker, A. M. Riley, S. I. Smedley and F. L. Tanzella, Proceedings of ICCF3, 5 (1993)

15. K. Kunimatsu, N. Hasagawa, A. Kubota, N. Imai, M. Ishikawa, H. Akita and Y. Tsuchida, Proceedings of ICCF3, 31 (1993)

16. A. Takahashi, M. Ohta and T. Mizuno, Jpn. J. Appl. Phys., 40, 7031 (2001)

17. K. Kamada, H. Kinoshita and H. Takahashi, Jpn. J. Appl. Phys., 35, 738 (1996)

18. H. Numata, R, Takagi, I. Ohno, K. Kawamura and S. Haruyama, Proceedings of ACCF2, 71 (1991)

19. H. Numata and M. Ban, Proceedings of ICCF12 (2005)

20. T. Mizuno, Y. Toriyabe, A. Takahashi and A. Takada, Proceedings of JCF6, 19 (2005)

21. T. Mizuno and Y. Toriyabe, Proceedings of ICCF12 (2005)

22. G. H. Miley and J. A. Patterson, J. New Energy, Vol.1, No.3, 5 (1996) 